\title{
Prenatal risk factors and postnatal central nervous system function
}

\author{
Johannes Hebebrand · Frank Verhulst
}

Published online: 28 September 2014

(c) Springer-Verlag Berlin Heidelberg 2014

Risk factors prior to birth can be subdivided according to timing, duration, dosage of exposure, and their mode of action; these variables determine which tissues/organs in the offspring are affected and to what extent. The central nervous system (CNS) as the most complex human organ is frequently affected. Sometimes exposures cause gross damage to the brain, but subtle effects due to exposures to single and frequently multiple risk factors are much more common. Multiple risk factors with small effects are difficult to unravel and trace back to their individual effect.

In the preconception phase, the parental haploid genomes can harbor genetic polymorphisms and molecular genetic or chromosomal aberrations that are associated with variations in CNS functioning of the offspring. Depending on the developmental gene regulation, structural and/or functional damage to the CNS ensues pre or postnatally. The extent to which functionally relevant epigenetic alterations are transmitted via the germ lines is currently a subject of scientific debate. After conception, short or longer acting risk factors can affect brain function via diverse mechanisms including epigenetic modifications of specific genes. Drugs can cross the placenta and exert their detrimental effect by binding to the respective receptors in the developing brain; such receptors are

\footnotetext{
J. Hebebrand

Department of Child and Adolescent Psychiatry, Rheinische Kliniken, University of Duisburg-Essen, Virchowstr. 174, 45147 Essen, Germany

e-mail: johannes.hebebrand@uni-due.de

F. Verhulst $(\bowtie)$

Department of Child and Adolescent Psychiatry, Erasmus University Medical Center, Sophia Children's Hospital, P.O. Box 2060, 3000 CB Rotterdam, The Netherlands

e-mail: f.verhulst@erasmusmc.nl
}

frequently already expressed in the embryonic stage. The reduction of placental blood flow as a consequence of the exposure to specific risk factors-like alcohol, nicotine and/or cannabis use-is one example of a mechanism that may indirectly impact offspring CNS function.

The prenatal period typically refers to the time from conception to birth, during which a host of maternal exposures to for example drugs, medications, toxins, infectious agents, radiation as well as maternal psychological factors can directly or indirectly influence the offspring's brain structure and/or function. Due to the rapid development from zygote to term newborn within approximately 270 days, the timing at which an embryo (first 8 weeks after conception) or fetus is exposed to nongenetic risk factors is crucial; particularly early exposure can entail substantial CNS structural aberrations or even lead to intrauterine death. Maternal malnutrition and other factors associated with poverty represent examples of risk factors, which continuously impinge on the embryo/fetus. Unfortunately, it is not uncommon for multiple risk factors (genetic and non-genetic) to be present at the same time. Thus, pregnant women who drink alcohol frequently also smoke; their genetic makeup and social environment predispose them to stress, depression, violence and poor nutrition both prior to and during pregnancy. Such interrelated exposures to a range of risk factors entail serious methodological problems for determining the effects of any single factor; this is even more so the case, because the same risk factors can remain operative postnatally. According to the dual hit hypothesis, a risk factor during intrauterine development may entail functionally non-overt alterations, which require a second postnatal factor to result in an overt, perturbed function, such as a psychiatric disorder, later in life. The genome of the offspring can attenuate or aggravate the effect of a risk factor. 
It is readily evident that the pregnant mother's behavior and her physical and psychological health influence the development of her child. The father, however, plays a weaker and more indirect role with respect to prenatal environmental risk exposure. Apart from his contribution to the genetic makeup of the offspring, the father will influence the developing child via the maternal environment.

Current knowledge about the etiology of psychiatric disorders indicates that individual differences in child neurodevelopment arise from a large number of causal factors, both genetic and non-genetic, most of which contribute a relatively small effect. The effect sizes of any common genetic variant on child behavioral, emotional and cognitive functioning are likely to be very small. In addition, complex gene-environment interplay, including epigenetic modifications, may well account for the multifactorial etiology of psychiatric disorders. In this context it is important to point out that many prenatal risk factors are readily amenable to prevention strategies. Social conditions and political systems are highly relevant with respect to exposure to prenatal risk factors and their prevention. Malnutrition and environmental pollution are examples of serious risks that can and must be reduced. Smoking rates among adolescents and young adults have been reduced successfully over the past decades due to prevention efforts in many industrialized countries; these efforts must continue. Prenatal alcohol exposure represents a major and well known risk factor; we urgently require more stringent prevention strategies to minimize alcohol exposure during pregnancy. The political discussions in several countries related to a legalization of cannabis use by no means sufficiently take into account potential risks for the offspring of consuming young adults.

The frequency with which the prenatal period is endangered by risks is high; the consequences for the offspring may last a lifetime and can lead to substantial impairment. As clinicians and researchers, who deal with mental disorders in childhood and adolescence, we must recognize and act upon prenatal risk factors that cause or predispose to cognitive and/or emotional problems of future children. Who else is as keenly aware of the limitations inherent to postnatal treatment?

Taken together, these considerations render a special issue of European Child and Adolescent Psychiatry devoted to current knowledge of prenatal risk factors, their effects on brain structure/function, and their behavioral and cognitive implications, timely. The issue includes a total of ten reviews and original articles for a selected number of pre-pregnancy and prenatal risk factors to illustrate their overall importance and to provide an insight into potentially underlying mechanisms:

1. Prenatal alcohol exposure is one of the most prevalent, modifiable risk factors for physical (in particular neurological), behavioral, and cognitive problems in the offspring. In Great Britain, for example, $75 \%$ of females reported having consumed alcohol at least once during pregnancy. The most severe effect of prenatal alcohol exposure on offspring, the fetal alcohol syndrome, was described for the first time in 1968. It has subsequently become apparent that milder forms of this disorder exist which have been termed fetal alcohol spectrum disorders. These may affect up to 2-5\% of children in developed countries. As mental health professionals, we are particularly confronted with their problems including hyperactivity, impulsivity, inattention, reduced cognitive functioning, and conduct problems; upon careful physical and psychological examinations, features can be delineated that help to distinguish these children from those with attention deficit/ hyperactivity disorder and conduct disorders not related to prenatal alcohol exposure. It is currently unknown to what extent low levels of alcohol exposure in utero impinge on behavioral and cognitive functioning of a child; on the other hand, particularly maternal binge drinking entails substantial risks as a result of high blood alcohol concentrations. Polymorphisms in the offsprinǵs genes for alcohol dehydrogenases predispose to lower IQ as a consequence of prenatal alcohol exposure through specific gene-environment interactions. Prenatal alcohol exposure exerts its negative effects via different mechanisms which include direct effects on the offspring's central nervous system via passage through the placenta and a reduced utero-placental blood flow [1].

2. Furtado et al. [2] identified socio-demographic, psychological and social maternal risk factors associated with fetal alcohol spectrum disorders in their systematic literature review. Mothers of affected children tended to be older, less educated, and more frequently had relatives with alcohol use disorders. Importantly, older siblings of children with fetal alcohol spectrum disorders may also be affected. This finding stresses the need for early identification of fetal alcohol spectrum disorders to prevent prenatal alcohol exposure in subsequent pregnancies.

3. Sayal et al. [3] studied the effects of episodic maternal binge drinking during pregnancy on both mental health and academic outcomes in offspring at age eleven in the Avon Longitudinal Study of Parents and Children. Eighty-five percent of all pregnant females resident in the Avon area with an expected delivery date between April 1991 and December 1992 agreed to participate in the study. Questionnaires filled in at gestation week 18 were used to determine maternal 
alcohol consumption during the first trimester; at 32 weeks of gestation more than half of the women again provided information on alcohol intake during pregnancy. At both 18 and 32 weeks the number of days during which at least four units of alcohol had been consumed over the last 4 week period were assessed. In addition, the frequency of drinking was also assessed thus allowing adjustment to determine effects of binge drinking. Child mental health was measured with the Strengths and Difficulties Questionnaire; academic outcomes were operationalized by use of standardized, age-adjusted total scores of the school examination taken during the final year at primary school. The analyses were adjusted for both maternal and offspring confounders including maternal mental health, preterm birth, and the child's gender and birth weight. A quarter of mothers reported drinking $\geq$ four units of alcohol at least once during pregnancy. A binge pattern of drinking as a risk factor independent of daily drinking in mothers was associated with higher levels of hyperactivity and inattention and reduced academic outcomes in their children at age 11 .

4. In the study of Knudsen et al. [4] the authors attempted to dissect effects of maternal pre-pregnancy drinking from those induced by drinking after conception on toddler problems. The authors used data of the Norwegian Mother and Child Cohort study, which included information on 56,682 motherchild dyads. Mothers answered questions on prepregnancy risk drinking at gestation week 17/18; alcohol use during pregnancy was assessed at weeks 18 and 36. Problems of the offspring were assessed with the Child Behavior Checklist at ages 18 and 36 months. Maternal pre-pregnancy risk drinking predicted toddler internalizing and externalizing problems at both ages; the odds ratio for internalizing behavior was non-significantly higher at 36 than at 18 months (odds ratios 1.7 and 1.5, respectively); the odds ratios for externalizing behavior were approximately 1.6 for both time points. However, in the model adjusted for all covariates including maternal anxiety, ADHD, and importantly pre and postnatal alcohol consumption the effect of pre-pregnancy risk drinking on only internalizing behavior at age 36 months remained significant. The authors critically discuss that it is unclear if pre-pregnancy drinking is an independent risk factor or a marker of genetic and environmental characteristics which increase the risk for toddler behavioral problems.

5. In comparison to the well-established effects of prenatal alcohol exposure on mental health, the effects of intrauterine exposure to nicotine are less clear [5]. European epidemiological studies indicate that between 10 and $38 \%$ of females smoke during pregnancy. Rates of pregnancy complications are elevated among female smokers. Active maternal smoking is known to result in a host of adverse effects on the offspring including reduced fetal growth, reduced birth weight, sudden infant death, and respiratory problems. Nevertheless, the effects on offspring mental health are much less clear. Possibly due to variations in statistically controlling for confounders, including maternal age, educational level, and socio-economic status, some studies have reported an association between prenatal smoking and children's intelligence, academic functioning, or attention problems, whereas others have not. Rodent studies, too, have not come up with consistent findings on cognition or behavior. Taken together, these findings cast some doubt on the causal role of smoking on mental problems in the offspring.

6. In addition to alcohol and nicotine use, cannabis represents another risk if used during pregnancy. Calvigioni et al. [6] describe the variations in the legal situation regarding cannabis use in different countries and note that its popularity in young adults is rising. Consequently, cannabis use by pregnant women is increasing with currently about $10 \%$ of pregnant women in the US and Europe using cannabis. Research focusing on cannabis-induced fetal developmental risks is just in its infancy, and studies are scarce. Negative developmental impact of prenatal cannabis exposure has been studied in three major prospective longitudinal studies revealing reduced birth weight and head circumference in addition to other effects. Observations like an increased fetal pulsatility index and resistance index of the uterine artery may point to diminished oxygen and nutrient accessibility as one potential mechanism for growth retardation. Some evidence suggests that an impaired cognitive development may result from prenatal cannabis exposure; however, similar to the more numerous studies related to prenatal exposure to cigarette smoking it is difficult to establish causality in light of the numerous confounders. Animal studies including in-depth investigations of cannabinoid signaling mechanisms suggest that delta-9-tetrahydrocanabinol (THC; the main psychoactive component of cannabis) induced receptor desensitization and downregulation renders a potential mechanism for the negative effects of cannabis use.

7. In their review, Beijers et al. [7] take a look at putative mechanisms involved in fetal programing via maternal stress during pregnancy. It is thought that the respective epigenetic alterations may even be 
transmitted from the offspring to the subsequent generation. The concept of prenatal psychosocial stress includes the stressors, their appraisals by the future mother and her stress responses including emotional reactions. The role of the hypothalamuspituitary-axis stands out in this field of research. High maternal cortisol levels can lead to elevated fetal cortisol concentrations, which are assumed to have a negative effect on the development of amygdala, hippocampus and prefrontal cortex. Other putative mechanisms via which maternal stress during pregnancy can influence offspring cognition and behavior include effects on the maternal immune system with subsequent vulnerability to infections, increased maternal catecholamine concentrations which can restrict placental and fetal blood flow, and finally via the colonization of the gut microbiome of the newborn. Animal models have revealed cognitive impairments and behavioral, immunologic, and metabolic alterations in offspring of dams exposed to stress during pregnancy. In humans, preterm birth and low birth weight are potential outcomes of in utero exposure to maternal stress. Attention deficit/hyperactivity, increased anxiety, and in case of severe exposure psychosis and depression have been reported to be associated with prenatal stress; further research is warranted to establish causality.

8. In contrast to the effects of maternal postpartum depression on child development, the effects of maternal depression during pregnancy on children's later functioning have been studied less frequently. During the first and second/third trimesters, between 7 and 11 and 9-13\%, respectively, of females are depressed. Maternal depression during pregnancy is thought to be responsible for an activation of the hypothalamus-pituitary-adrenal axis and the autonomic nervous system of the fetus with potential implications for later child emotional and behavioral functioning. A major methodological limitation of studies attempting to assess these outcomes is that, in most studies, mothers report both on their own depression and on the behavior of their child, increasing the likelihood of halo effects in ratings, reflecting shared method variance. In addition, genetic effects and postnatal maternal psychopathology are potential confounders in such studies. Despite these inherent methodological issues, there is evidence to suggest that depression during pregnancy is associated with an elevated risk for offspring antisocial behavior [8]. Because antenatal depression is a marker for multiple biological and psychosocial risks, both recognition and treatment of maternal depression during pregnancy warrant further studies.
9. Given the potentially negative effects of maternal depression during pregnancy on the offspring, physicians are confronted with the question as to whether or not prescribe antidepressants. Current knowledge of risks associated with in utero exposure to antidepressants and anxiolytics is summarized by El Marroun et al. [9] in a systematic review. Between one and eleven percent of females use antidepressants or anxiolytics during pregnancy; their use is seemingly increasing, particularly because of females on maintenance antidepressant treatment to prevent relapse. Again, the disentanglement of the effects induced by antidepressants or anxiolytics from those of maternal psychopathology and associated factors such as elevated rates of smoking and alcohol consumption, less prenatal care, and poor self-care, proves to be difficult. Antidepressants and anxiolytics pass the placental barrier and may thus affect fetal development. Animal studies indicate that intrauterine exposure to such medications affects neurotransmitter systems and may have long lasting implications. In humans, congenital anomalies including neural tube and cardiovascular defects have been observed infrequently in offspring exposed to serotonin reuptake inhibitors (SSRI). However, the causal role of SSRIs is unclear. Some studies suggest a causal role of antidepressants and anxiolytics on motor development, internalizing problems and autism. However, systematic randomized case-control studies are required to test the causality of prenatal exposure to these substances on child development. The translation of the currently available results into clear-cut clinical recommendations is challenging.

10. In an original article, Kvalevaag et al. [10] looked into the effects of paternal psychological distress at weeks 17 or 18 of gestation on physically aggressive behavior of the offspring. This study was also based on the Norwegian Mother and Child Cohort study and included information on 19,580 father-child dyads. Paternal mental health during the past 2 weeks was assessed via self-report upon use of the Symptom Checklist-5, which includes five items reflecting paternal distress. One item of the Child Behavior Checklist ("Hits others") completed by the mother at ages 18, 36, and 60 months of their child was used as outcome. Approximately $16 \%$ of the children were reported to hit others at age five. After adjustment for several covariates including socio-economic status, paternal cigarette and alcohol use, fathers' marital satisfaction, and mothers' psychological distress, only a trend for a higher risk of children hitting at 5 years was detected if fathers had a high level of psychological distress (odds ratio: 1.24; $p=0.1$ ). 
Stratification for gender revealed that this effect applied to the girls (OR: $1.46 ; p<0.05$ ) only (OR for boys: 1.03).

This issue shows that across the multitude of studies, that have been performed on the effects of the prenatal environment, exposures to maternal alcohol use, smoking, cannabis or SSRI use, and maternal depression, anxiety and family stress have all been associated with less fetal growth including that of the brain. In contrast, a number of studies showed that maternal exposures, which negatively affect intrauterine growth, had little, if any, impact on later child development if carefully controlled for confounding. How can we reconcile these findings?

For one, postnatal "catch-up" growth in combination with the plasticity of the maturing brain may prevent the emergence of functional implications. Indeed, the findings of a number of studies support the view that several negative effects of prenatal environmental exposures that are not extreme can be compensated later in life. The negative prenatal risk factors could thus be viewed as resulting in a merely transient indentation. In such a case, the few reported associations of prenatal environmental exposures with later problem behaviours or reduced cognition would suggest that much of the observed intrauterine association is due to genetic confounding or spill over of parental behaviour from the prenatal to the postnatal period. As such, associations between various single prenatal exposures and later child problems can be used as a marker for risk, since the overall effect of a combination of multiple prenatal and early postnatal environmental, including parenting risks such as harsh parenting, put young children at risk for life-long behavioral, cognitive and social problems.

On the other hand, more caution may well be warranted. Alcohol related research has taught us that dosage is important. There is no doubt, that an extreme prenatal exposure can entail long lasting functional implications. In contrast, we do not have sufficient evidence to exclude small effects of low exposures. A threshold for alcohol, if it exists at all, may depend on both maternal and offspring features apart from timing and thus vary individually. Accordingly, to establish the causal role of a given risk factor on longer term development, it may be prudent to focus on cases with high prenatal exposures, obviously entailing the inherent difficulty of ascertaining a sufficiently large sample size.

The adjustments made in an effort to exclude the effects of confounders may also be problematic, because statistical power to detect an association is reduced. In addition, it may be combinations of different risk factors that entail an elevated risk. Very large sample sizes are a prerequisite for addressing these complex issues.

Finally, we may have to take a more long term perspective. We are typically dealing with studies of younger children. It cannot be excluded that prenatal risk factors induce an elevated vulnerability for mental problems throughout life. If the second hit hypothesis is an indicator for the complexity of such interactions, it may well be necessary to expand studies aiming to assess the functional implications of (potential) prenatal risk factors to adolescents and young adults.

In conclusion, we definitely need more research to further elucidate prenatal risk factors. Apart from large-scaled longitudinal studies, we should consider studies of motherchild dyads who have received a high dosage of a particular risk factor. The emerging field of epigenetics reveals how crucially we depend on an insight into the underlying molecular mechanisms. We have hardly begun to address gene-risk factor interactions. For the assessment of prenatal risk factors we need to look at the genetic make-up of both the mother and the child. Animal studies must be pursued to obtain hypotheses as to the relevance, importance of timing and mode of action of specific risk factors. A more general approach for animal studies may relate to the analysis of the implications of a reduced fetal (brain) growth on later behavior and cognition.

\section{References}

1. Dörrie et al (2014) Fetal alcohol spectrum disorders. Eur Child Adolesc Psychiatry. doi:10.1007/s00787-014-0571-6

2. Furtado et al (2014) Identifying maternal risk factors associated with fetal alcohol spectrum disorders: a systematic review. Eur Child Adolesc Psychiatry. doi:10.1007/s00787-014-0603-2

3. Sayal et al (2014) Prenatal exposure to binge pattern of alcohol consumption: mental health and learning outcomes at age 11. Eur Child Adolesc Psychiatry. doi:10.1007/s00787-014-0599-7

4. Knudsen et al (2014) Maternal pre-pregnancy risk drinking and toddler behavior problems: the norwegian mother and child cohort study. Eur Child Adolesc Psychiatry. doi:10.1007/s00787014-0588-x

5. Tiesler and Heinrich (2014) Prenatal nicotine exposure and child behavioural problems. Eur Child Adolesc Psychiatry. doi:10. 1007/s00787-014-0615-y

6. Calvigioni et al (2014) Neuronal substrates and functional consequences of prenatal cannabis exposure. Eur Child Adolesc Psychiatry. doi:10.1007/s00787-014-0550-y

7. Beijers et al (2014) Mechanisms underlying the effects of prenatal psychosocial stress on child outcomes: beyond the HPAaxis. Eur Child Adolesc Psychiatry. doi:10.1007/s00787-0140566-3

8. Waters et al (2014) Antenatal depression and children's developmental outcomes: potential mechanisms and treatment options. Eur Child Adolesc Psychiatry. doi:10.1007/s00787-014-0582-3

9. El Marroun et al (2014) Maternal use of antidepressant or anxiolytic medication during pregnancy and childhood neurodevelopmental outcomes: a systematic review. Eur Child Adolesc Psychiatry. doi:10.1007/s00787-014-0558-3

10. Kvalevaag et al (2014) Does paternal mental health in pregnancy predict physically aggressive behavior in children? Eur Child Adolesc Psychiatry. doi:10.1007/s00787-014-0587-y 